Freemantle, N., House, A., Song, F., et al (1994) Prescribing selective serotonin reuptake inhibitors as strategy for prevention of suicide. British Medical Journal, 309, 249-253.

Jönsson, B. (1994) Letter. Health Economics, 3, 305-307.

KIND, P. \& Sørensen, J. (1993) The cost of depression. International Clinical Psychopharmacology, 7, 191-195.

Montgomery, S. A., Henry, J., MCDonald, G., et al (1994) Selective serotonin reuptake inhibitors: meta-analysis of discontinuation rates. International Clinical Psychopharmacology, 9, 47-53.

Stoudemire, A., Frank, R., Hedemark, N., et al (1986) The economic burden of depression. General Hospital Psychiatry, 8, 387-394.

Stockholm School of Economics

B. JöNSSON

Box 6501, S-11383 Stockholm

Sweden

Institute of Psychiatry

P. BEBbington

London SE5 8AF

\section{Tourette's syndrome and psychiatric disorders}

SIR: Pauls et al (BJP, February 1994, 164, 215-221) claim to provide evidence against our proposal (Comings \& Comings, 1987; Comings, 1995a,b) that depression and anxiety disorders are genetically related to the $T S$ (Gts) gene(s). In reality their report provides strong evidence in favour of such a relationship.

Pauls et al have consistently maintained that the wide range of psychiatric disorders comorbid to Tourette's syndrome (TS) are all due to proband ascertainment bias, and would not be increased in frequency in the relatives of TS probands. Now they find that in fact the frequency of generalised anxiety disorder, major depressive disorder, panic disorder, simple and social phobia are significantly increased in frequency in 338 relatives of TS probands compared with 113 relatives of controls (Table 2). To extricate themselves from this turn of events, they now retreat to the extraordinary position of claiming that these disorders are unrelated to the Gts genes because their frequency is not significantly higher in first degree relatives without TS, chronic tics (CT) or obsessive-compulsive disorder (OCD) compared with relatives of controls. However, their own studies state that TS is inherited as an autosomal dominant trait with $100 \%$ penetrance in males, and when OCD is included, $71 \%$ penetrance in females (Pauls \& Leckman, 1986). Thus, only $15 \%$ of relatives of TS probands without TS, CT or OCD should be carrying the Gts genes. As such, one would not be able to identify any of the pliotrophic effects of the Gts genes in a group of subjects where so few carry the genes. This would be analagous to attempting to claim that the Huntington's disease gene does not cause dementia because none of the relatives without HD show dementia.

They next claim that the increased frequency of these behaviours in the relatives that they observed was not due to the Gts gene(s), but was instead due to the presence of OCD. Again they seem to have forgotten their own paper (Pauls \& Leckman, 1986) showing that the Gts gene(s) can cause OCD with or without tics. Thus, they would accept that Gts genes cause OCD, and that OCD produces depression and anxiety disorders, but deny the whole equation that $G$ ts genes can cause depression and anxiety disorders. The demonstration by Pauls et al, of the increased frequency of associated behaviours in relatives with OCD but no tics, totally supports our suggestion that some individuals in the general population without tics may have these conditions because they carry Gts genes.

Commgs, D. E. (1995a) Genetic factors in depression based on studies of Tourette's syndrome and attention deficit hyperactivity disorder probands and relatives. American Journal of Medical Genetics (in press).

- (1995b) Tourette's syndrome: a hereditary neuropsychiatric spectrum disorder. Annals of Clinical Psychiatry (in press).

- \& Comngs, B.G. (1987) A controlled study of Tourette's syndrome. I-VII. American Journal of Human Genetics, 41, 701-866.

Pauls, D. L. \& Leckman, J. F. (1986) The inheritance of Gilles de la Tourette's syndrome and associated behaviors. Evidence for autosomal dominant transmission. New England Journal of Medicine, 315, 993-997.

Department of Medical Genetics

D. E. Comings

City of Hope Medical Center

Duarte, CA 91010

USA

\section{ECT seizure duration and efficacy}

SIR: The study by Fear et al (BJP, October 1994, 165, 506-509), their prior correspondence (BJP, March 1993, 162, 421-423), and two previous studies (Mitchell et al, 1991; Malsch et al, 1992) resurrect the issue of whether ECT seizure duration per se is related to the antidepressive efficacy of ECT. For example, Malsch et al (1992) state that "one of the unsolved questions of [ECT] is the effect of seizure duration on the efficacy of treatment".

The notion that ECT seizure duration might be related to its antidepressive efficacy has been advanced by the works of Maletzky (1978) and Kramer (1983). However, neither investigation demonstrated a significant positive correlation 
between the antidepressive efficacy and seizure duration without confounding seizure duration with the actual number of ECT treatments administered. However, because existing studies comparing methohexitone (Malsch et al, 1992; Fear et al) or thiopentone (Mitchell et al, 1991) to propofol anaesthesia have found no seizure duration effect with the same number of treatments, one must conclude that seizure duration is irrelevant to the antidepressive efficacy. Furthermore, no other well-controlled ECT study to date has demonstrated a relation between seizure duration and antidepressive efficacy.

What appears to be important with respect to therapeutic efficacy, as discussed elsewhere (BJP, May 1983, 142, 536-537), is not the precise duration of seizure activity, but rather the administration of 5-12 adequately generalised tonic-clonic seizures of at least 20 seconds duration (American Psychiatric Association, 1990). In this regard, two points should be made. Firstly, in the two studies comparing propofol with methohexitone anaesthesia (Malsch et al, 1992; Fear et al), adequate numbers of seizures were administered (approximate mean number of treatments across studies: 7.7 (methohexitone) and 7.3 (propofol)) which were of adequate duration (mean seconds of seizure activity: 46.9 (methohexitone) and 37.1 (propofol)).

Secondly, electrically induced seizures may vary in degree of generalisation (BJP, May 1983, 142, 536-537). More precisely, seizure generalisation may increase in a curvilinear fashion (i.e. quadratic curve) until an asymptote ("ceiling effect") is reached with respect to the amount of electrical energy delivered (Sackheim et al, 1993). When this asymptote is reached, the antidepressive efficacy of ECT has probably reached its maximum; seizure duration at this point is probably just a correlate of the causal relationship between seizure generalisation and electrical energy, the latter of which alleviates depression.

American Psychiatric Association (1990) The Practice of Electroconvulsive Therapy: Recommendations for Treatment, Training and Privileging. Washington, DC: APA.

KRAMER, B. A. (1983) Seizure parameters in depressed patients receiving electroconvulsive therapy: a pilot study. Comprehensive Psychiatry, 24, 259-261.

MAlETZKY, B. M. (1978) Seizure duration and clinical effect in electroconvulsive therapy. Comprehensive Psychiatry, 19, 541-550.

Malsch, E., Gratz, I. \& Mani, S. (1992) Efficacy of electroconvulsive therapy after propofol or methohexitone anesthesia. Anaesthesia and Analgesia, 74, S192.

Mrtchell, P., Torda, T., Hickie, L., et al (1991) Propofol as an anaesthetic agent for ECT: effect on outcome and length of course. Australian and New Zealand Journal of Psychiatry. 25, 255-261.
Sackhem, H., Prudic, J., Devanand, D. P., et al (1993) Effects of stimulus intensity and electrode placement on the efficacy and cognitive effects of electroconvulsive therapy. New England Journal of Medicine, 328, 839-846.

W. F. DANIEL

Department of Psychology

University of New Mexico

Logan Hall

Albuquerque, New Mexico

USA

AUTHORS REPLY: We thank Dr Daniel for his correspondence, which is broadly supportive of the points made in our paper. It is encouraging to see that the evidence concerning seizure duration and the efficacy of ECT continues to be critically appraised, since it is only through this that an understanding of the mechanisms underlying effective ECT will be ascertained. There are some points raised in the letter which merit further consideration.

There are significant problems in interpreting the literature with respect to the relationship between antidepressive efficacy and seizure duration. In addition to the error of combining the effects of seizure duration with those of treatment numbers so as to obscure the true effect of each, it is difficult to know how it would be possible to show, simply, a positive correlation between antidepressive efficacy and seizure duration. Clearly it is far easier to show statistically that two samples differ rather than to prove that they do not. There is, however, increasing evidence that seizure duration in current ECT practice is not clinically relevant so long as a seizure occurs. Perhaps useful progress could be made in attempting to define what constitutes a seizure, since existing literature is conflicting, and this would represent an important step in the standardisation of ECT research.

Daniel inadvertently illustrates this point in calculating mean seizure durations across two recent studies (Malsch et al, 1992; Fear et al). These figures are not directly comparable since they invoke different methods of measuring seizure duration. The first study used the purely motor manifestations of a seizure to measure its duration, using the "cuff technique", whereas in the second, both physical (unspecified method) and EEG durations were measured. In a third paper (Mitchell et al, 1991), physical measurement was performed "by an experienced psychiatric nurse without use of the 'cuff' (isolated arm) method". Under these circumstances, it is not valid to say that the seizures were of adequate duration since the comparability of their durations is uncertain. 